\title{
Ações de promoção da saúde nas estratégias saúde da família
}

\author{
Health promotion actions in family health strategies
}

\author{
Betina Brixner, ${ }^{1}$ Carine Muniz, Jane Dagmar Pollo Renner, ${ }^{1}$ Hildegard Hedwig Pohl, \\ Edna Linhares Garcia, ${ }^{1}$ Suzane Beatriz Frantz Krug' \\ 'Universidade de Santa Cruz do Sul (Unisc), Santa Cruz do Sul, RS, Brasil.
}

Recebido em: 03/10/2017 / Aceito em: 21/12/2017 / Publicado em: 30/12/2017

betinabrixner@yahoo.com.br

\section{RESUMO}

Objetivo: considerando a necessidade de uma intensificação dos processos de educação em saúde no território brasileiro, o objetivo deste estudo foi identificar ações de promoção da saúde desenvolvidas na "Estratégia Saúde da Família", em estudos científicos publicados nos últimos dois anos, tendo em vista a publicação da Portaria $n^{\circ}$ 2.446, que redefiniu a Política Nacional de Promoção da Saúde. Método: foi realizada uma revisão da literatura em duas bases de dados utilizando os descritores "Promoção de Saúde", "Atenção Primária à Saúde" e "Estratégia Saúde da Família". Resultados: foram encontrados 29 artigos; 16 indexados no SCIELO e 13 no LILACS. Após revisão e análise dos artigos foram incluídos sete estudos. Os principais resultados referem-se a ações voltadas para a população por meio de orientações em geral, utilizando como ferramenta principal palestras e atividades em grupo. Estas ações tiveram como objetivo conscientizar a população sobre alimentação saudável, prática de atividades físicas, prevenção de doenças sexualmente transmissíveis, uso de álcool, tabaco e outras drogas, além de orientações sobre saúde sexual e reprodutiva. Considerações finais: as equipes multiprofissionais das Estratégias Saúde da Família organizam ações de promoção, na perspectiva da educação em saúde, conforme preconizado nos "temas prioritários" da Política Nacional de Promoção da Saúde. Nota-se também que algumas dessas equipes multiprofissionais desenvolvem atividades de maneira contínua, enquanto outras em período específico. Reforça-se a necessidade da realização de ação prática e oficinas que visem promover saúde e melhorar o estilo de vida da população.

Palavras-chave: Promoção da Saúde; Atenção Primária à Saúde; Estratégia Saúde da Família; Educação em Saúde.

\section{ABSTRACT}

Objective: considering the necessity for an intensification of the processes of health education in the Brazilian territory, the objective of this study was to identify the health promotion actions developed in the "Family Health Strategy", on scientific studies published at last 2 years, in view of a publication of an Ordinance $n^{\circ}$ 2.446, which redefined the National Policy for Health Promotion. Method: a review of the literature was carried out in two databases using 3 descriptors: "Health Promotion", "Primary Health Care" and "Family Health Strategy". Results: the review has found 29 articles; 16 of them were indexed in the SCIELO database and 13 others in the LILACS database. After a review and analysis of the articles, seven studies were included. The main results refer to actions directed to the population through orientations in general, using as main tool lectures and group activities. These actions aimed to raise awareness among the population about healthy eating, physical activity, prevention of sexually transmitted diseases, use of alcohol, tobacco and other drugs, as well as guidelines on sexual and reproductive health. Closing remarks: the multiprofessional teams of the Family Health Strategies organize promotion actions, from the perspective of health education, as recommended in the "priority themes" of the National Policy for Health Promotion. It is also noted that some of these multiprofissional teams carry out activities in a continuous manner, while others in a specific period. It reinforces the need for practical action and workshops aimed at promoting health and improving the population's lifestyle.

Keywords: Health Promotion; Primary Health Care; Family Health Strategy; Health Education. 


\section{INTRODUÇÃO}

Com a construção e implementação da Política Nacional de Promoção da Saúde - PNPS (instituída pela Portaria MS/GM n 687, de 30 de março de 2006) observa-se mudanças nos "modos de organização, planejamento, realização, análise e avaliação do trabalho na área da saúde ${ }^{\prime 1}{ }^{1}$ Ao construir ações de promoção da saúde, o objetivo deve estar centrado em promover a qualidade de vida, incluindo condições de trabalho, moradia, lazer, cultura, educação; em minimizar os riscos à saúde e a vulnerabilidade, bem como possibilitar o acesso a bens e serviços básicos. ${ }^{1,2}$

Estas ações de promoção da saúde também fazem parte das atribuições da atenção básica à saúde, que se configura como "um conjunto de ações, individuais e coletivas, que envolve a promoção e a proteção da saúde, a prevenção de doenças e agravos, a redução de danos, o diagnóstico, o tratamento, a reabilitação e a manutenção da saúde". ${ }^{3}$ Para Norman e Tesser, ${ }^{4}$ as medidas de promoção da saúde e prevenção de doenças devem ser incorporadas de forma complementar na organização do processo de trabalho das equipes da atenção básica.

Tendo em vista a necessidade de reorganizar a atenção básica de saúde, em 1994 (com base nos princípios do Sistema Único de Saúde - SUS) foi criada a Estratégia Saúde da Família (ESF), considerada como estratégia para expandir, qualificar e consolidar a atenção básica, para possibilitar a ampliação e aprofundamento dos princípios da atenção primária com o intuito de elevar a resolução dos problemas de saúde e o impacto na situação de saúde no âmbito individual e coletivo. ${ }^{3,5}$

Dentre as ações que podem ser desenvolvidas no campo da promoção da saúde, a PNPS aponta alguns temas prioritários evidenciados desde 2006, entre os quais estão:

Formação e educação permanente; Alimentação adequada e saudável; Práticas corporais e atividades físicas; Promoção da mobilidade segura; Promoção da cultura da paz e dos direitos humanos; Promoção do desenvolvimento sustentável Enfrentamento ao uso do tabaco, uso abusivo de álcool e outras drogas (p.22-23). ${ }^{2}$
Diante das considerações em relação à promoção da saúde, o estudo tem como objetivo identificar as ações de promoção da saúde desenvolvidas na ESF, em estudos científicos publicados nos anos de 2015-2016, no contexto brasileiro, considerando que a Portaria $n^{\circ}$ 2.446, de 11 de novembro de 2014, redefiniu a Política Nacional de Promoção da Saúde do Ministério da Saúde.

\section{MÉTODO}

Foi realizada uma pesquisa por meio de revisão de literatura a fim de discutir e ampliar os conhecimentos sobre as ações de Promoção da Saúde desenvolvidas nas ESFs. Os artigos foram identificados por meio de uma pesquisa na base de dados SCIELO e LILACS. A pesquisa foi realizada no mês de abril de 2017, utilizando as palavras-chaves "Promoção de Saúde"; "Atenção Primária à Saúde"; "Estratégia Saúde da Família", termos pesquisados e disponíveis nos Descritores em Ciências da Saúde (DeCS).

Como critérios de inclusão foram observados o período de publicação (2015 e 2016), estudos realizados no contexto brasileiro, idioma português e disponibilidade do texto completo nas bases de dados. Foram excluídos os artigos que apresentaram duplicidade, assuntos que não correspondiam ao tema pesquisado, os que não descreviam práticas como sendo estratégias de promoção da saúde e artigos de revisão. A discussão foi articulada através das ações desenvolvidas nas ESFs priorizando a Política Pública de Promoção da Saúde na Atenção Básica.

\section{RESULTADOS}

Foram selecionados 29 artigos, sendo que 16 destes estavam indexados na base de dados SCIELO e 13 no LILACS. Após as análises, foram incluídos sete artigos no presente estudo (Figura 1).

Os dados dos artigos incluídos neste estudo foram organizados de acordo com a autoria, ano de publicação, título, objetivo, tipo de estudo, tamanho amostral e estratégias de promoção da saúde (Quadro 1).

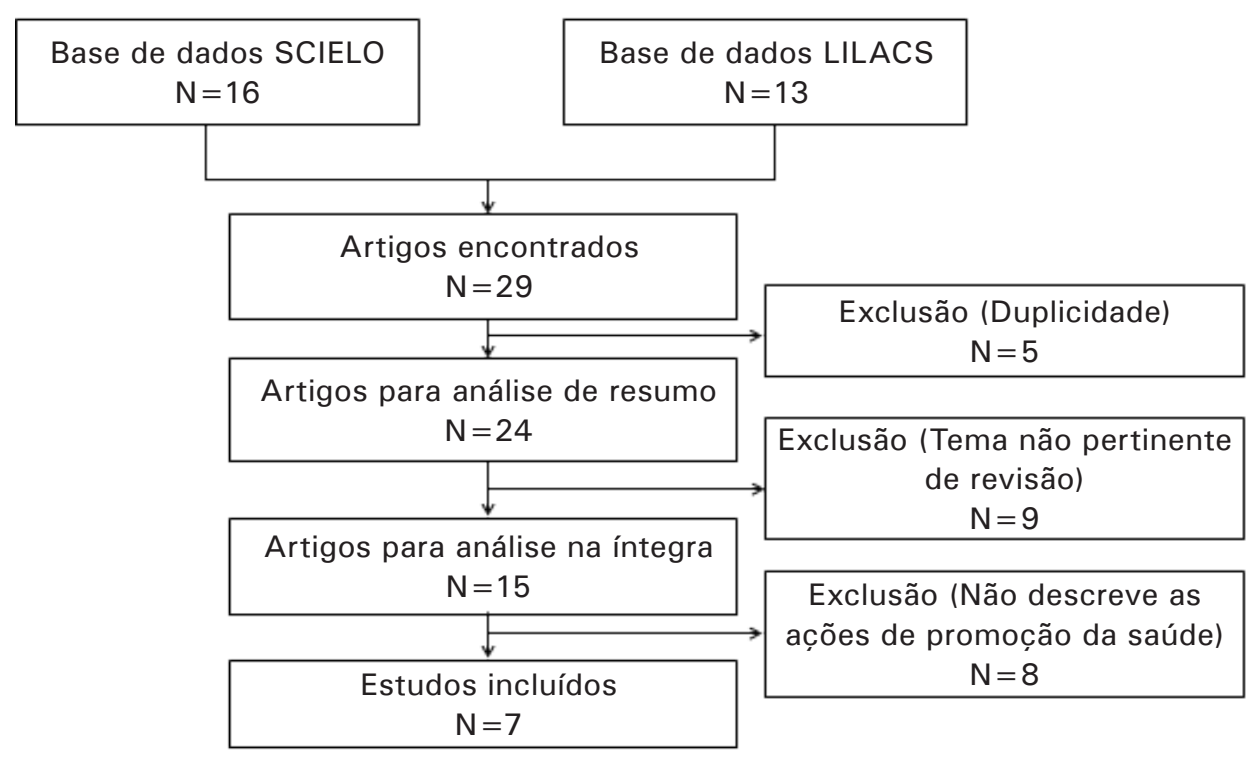

Figura 1 - Fluxograma da seleção dos artigos. 
Quadro 1 - Síntese de informações e ações de Promoção da Saúde contidos nos artigos inclusos no estudo.

\begin{tabular}{|c|c|c|c|c|c|}
\hline Autor (Ano) & Título & Objetivo & Tipo de Estudo & Tamanho Amostral & Ações de Promoção da Saúde Identificadas \\
\hline $\begin{array}{l}\text { Ferreira Neto et al. } \\
(2016)^{6}\end{array}$ & $\begin{array}{l}\text { Integralidade, condições de oferta } \\
\text { de serviços e processo de trabalho } \\
\text { de Equipes de Saúde da Família em } \\
\text { Belo Horizonte. }\end{array}$ & $\begin{array}{l}\text { Analisar a relação entre as ações de integrali- } \\
\text { dade e as condições de oferta dos serviços } \\
\text { e o processo de trabalho das Equipes da Es- } \\
\text { tratégia Saúde da Família (EqSF), em } 20 \text { UBS } \\
\text { de um distrito sanitário, no município de Belo } \\
\text { Horizonte (MG). }\end{array}$ & $\begin{array}{l}\text { Estudo } \\
\text { transversal }\end{array}$ & $\begin{array}{l}538 \text { profissionais das } \\
\text { equipes da ESF. }\end{array}$ & $\begin{array}{l}\text { Orientações gerais e sobre como utilizar os medicamen- } \\
\text { tos; levantamento do histórico de saúde do usuário; } \\
\text { orientações sobre hábitos de vida; escutas aos usuários } \\
\text { quanto à resistência às orientações e prescrições; en- } \\
\text { caminhamento para atividades de prevenção/promoção de } \\
\text { saúde (NASF; caminhadas; Lian Gong; academia da cidade; } \\
\text { atividades de lazer e comunitárias; grupos de saúde). }\end{array}$ \\
\hline $\begin{array}{l}\text { Vieira; Vieira } \\
(2016)^{7}\end{array}$ & $\begin{array}{l}\text { Saúde do idoso e execução da } \\
\text { Política Nacional da Pessoa Idosa } \\
\text { nas ações realizadas na atenção } \\
\text { básica à saúde. }\end{array}$ & $\begin{array}{l}\text { Identificar se os municípios que compõem a } \\
\text { Associação dos Municípios da Região Carbo- } \\
\text { nífera (AMREC) do estado de Santa Catarina } \\
\text { têm conhecimento e aplicam a PNSPI durante } \\
\text { as ações realizadas na atenção básica à } \\
\text { saúde, em especial nas Unidades de Estratégia } \\
\text { de Saúde da Família (UESF), à luz do que } \\
\text { dispõem a CF/88 e o Estatuto do Idoso. }\end{array}$ & $\begin{array}{l}\text { Estudo transversal } \\
\text { qualitativo e } \\
\text { descritivo }\end{array}$ & $\begin{array}{l}11 \text { coordenadores } \\
\text { da atenção básica à } \\
\text { saúde. }\end{array}$ & $\begin{array}{l}\text { Na Semana do Idoso ocorrem palestras temáticas } \\
\text { sobre prevenção de quedas, sexualidade e saúde bucal. } \\
\text { Orientação de saúde à população através de veículo de } \\
\text { comunicação (rádio). Orientações nos encontros de grupo } \\
\text { (Hiperdia). }\end{array}$ \\
\hline $\begin{array}{l}\text { Leite et al. } \\
(2016)^{8}\end{array}$ & $\begin{array}{l}\text { Estratégia Saúde da Família versus } \\
\text { centro de saúde: modalidades de } \\
\text { serviços na percepção do usuário. }\end{array}$ & $\begin{array}{l}\text { Compreender a percepção do usuário sobre } \\
\text { o processo de transição das modalidades de } \\
\text { serviços centro de saúde para ESF. }\end{array}$ & $\begin{array}{l}\text { Estudo transversal } \\
\text { qualitativo }\end{array}$ & $\begin{array}{l}12 \text { usuários do serviço } \\
\text { de saúde. }\end{array}$ & $\begin{array}{l}\text { Reuniões de grupo e palestras (hipertensão e diabetes); } \\
\text { Planejamento mensal para diabéticos; Visitas domiciliares. }\end{array}$ \\
\hline $\begin{array}{l}\text { Silva et al. } \\
(2016)^{9}\end{array}$ & $\begin{array}{l}\text { Uso de serviços de saúde por } \\
\text { diabéticos cobertos por plano pri- } \\
\text { vado em comparação aos usuários } \\
\text { do Sistema Único de Saúde no } \\
\text { Município de Belo Horizonte, Minas } \\
\text { Gerais, Brasil. }\end{array}$ & $\begin{array}{l}\text { Comparar indicadores de uso de serviços de } \\
\text { saúde e comportamentos em saúde entre } \\
\text { indivíduos que sabem ser portadores de dia- } \\
\text { betes e que são afiliados a planos privados de } \\
\text { saúde em relação aos seus pares usuários do } \\
\text { sistema público de saúde. }\end{array}$ & Estudo transversal & 370 sujeitos & $\begin{array}{l}\text { Orientações sobre hábitos saudáveis (tabagismo, con- } \\
\text { sumo de álcool, atividades físicas e/ou hábitos alimenta- } \\
\text { res). Apontam como desafio a realização de ações mais } \\
\text { efetivas para a prática de atividade física e reorganização } \\
\text { do sistema, a fim de melhorar o atendimento e desem- } \\
\text { penho da atenção primária de saúde. }\end{array}$ \\
\hline $\begin{array}{l}\text { Lindemann; } \\
\text { Mendoza-Sassi } \\
(2016)^{10}\end{array}$ & $\begin{array}{l}\text { Orientação para alimentação } \\
\text { saudável e fatores associados entre } \\
\text { usuários da atenção primária à } \\
\text { saúde no sul do Brasil. }\end{array}$ & $\begin{array}{l}\text { Descrever a prevalência de orientação para } \\
\text { alimentação saudável, diferenças entre } \\
\text { modelo assistencial e fatores associados entre } \\
\text { usuários da atenção primária de saúde. }\end{array}$ & Estudo transversal & $\begin{array}{l}1246 \text { usuários da } \\
\text { atenção primária }\end{array}$ & $\begin{array}{l}\text { Identificou-se precária orientação sobre alimentação sau- } \\
\text { dável. Os usuários que perceberam ter uma alimentação } \\
\text { inadequada foram os que receberam orientações dos } \\
\text { profissionais de saúde sobre este hábito. }\end{array}$ \\
\hline $\begin{array}{l}\text { Machado et al. } \\
(2015)^{11}\end{array}$ & $\begin{array}{l}\text { Programa saúde na escola: estraté- } \\
\text { gia promotora de saúde na atenção } \\
\text { básica no Brasil. }\end{array}$ & $\begin{array}{l}\text { Identificar e descrever as ações desenvolvidas } \\
\text { pelas Equipes de Saúde da Família (ESF) do } \\
\text { Brasil no Programa Saúde na Escola (PSE). }\end{array}$ & Estudo transversal & $\begin{array}{l}17.202 \text { equipes de } \\
\text { Saúde da Família }\end{array}$ & $\begin{array}{l}\text { Dentre as ações desenvolvidas pela equipe, nas escolas, } \\
\text { estão: levantamento do número de escolares com alguma } \\
\text { necessidade de saúde; ações sobre alimentação sau- } \\
\text { dável e segura; promoção da atividade física e práticas } \\
\text { corporais; debate com os professores e capacitação para } \\
\text { trabalhar com educação em saúde; atividades temáticas } \\
\text { sobre saúde sexual e reprodutiva, prevenção de DST/ } \\
\text { AIDS, uso de álcool, tabaco e outras drogas. }\end{array}$ \\
\hline $\begin{array}{l}\text { Pasqual; Carvalhaes; } \\
\text { Parada } \\
\text { 2015) }\end{array}$ & $\begin{array}{l}\text { Atenção à saúde da mulher após } \\
\text { os } 50 \text { anos: vulnerabilidade pro- } \\
\text { gramática na Estratégia Saúde da } \\
\text { Família }\end{array}$ & $\begin{array}{l}\text { Avaliar o processo de atenção prestado a } \\
\text { mulheres a partir dos } 50 \text { anos de idade em } \\
\text { Unidade de Saúde da Família (USF), tomando- } \\
\text { se como referência o conceito de vulnerabili- } \\
\text { dade programática. }\end{array}$ & $\begin{array}{l}\text { Estudo } \\
\text { epidemiológico }\end{array}$ & 715 mulheres & $\begin{array}{l}\text { Atividades educativas em grupos, porém com baixa } \\
\text { frequência na população estudada. Em relação as ativi- } \\
\text { dades propostas para mulheres hipertensas e diabéticas, } \\
\text { houve reduzida participação, mesmo resultado negativo } \\
\text { observado com mulheres sem nenhuma doença prévia. }\end{array}$ \\
\hline
\end{tabular}

Legenda: ESF (Estratégia de Saúde da Familia); UBS (Unidade Básica de Saúde); DST (Doença Sexualmente Transmissível); AIDS (Síndrome da Imunodeficiência Adquirida); NASF (Núcleo de Apoio à Saúde da Familia). 


\section{DISCUS5ÃO}

A partir dos artigos revisados para o desenvolvimento do presente estudo, observa-se que as estratégias de promoção da saúde realizadas pelas ESFs elencam um conjunto de ações e atividades educativas. Estas compõem um método que visa capacitar os indivíduos e/ou grupos por meio do controle dos determinantes sociais de saúde e melhoria da qualidade de vida. ${ }^{13}$

O trabalho desenvolvido nas ESFs é realizado por uma equipe multiprofissional que atende um território delimitado, nas quais são priorizadas ações de aproximação da equipe de saúde com a população adstrita, acessibilidade aos serviços básicos oferecidos e continuidade da atenção. ${ }^{5}$ Neste cenário, a promoção da saúde nas ESFs deve reafirmar os princípios de integralidade e participação da população, instituídos pelo o Sistema Único de Saúde (SUS)..$^{14}$

Dentre as estratégias organizadas pelas ESFs para promover a saúde, verifica-se que a "orientação para a população" foi a ação mais citada nas bibliografias estudadas, tais como: orientações sobre alimentação saudável, prática regular de atividade física, saúde sexual e reprodutiva, prevenção de Doença Sexualmente Transmissível, uso de álcool, tabaco e outras drogas. A importância destas orientações pode ser observada no estudo de Costa et al., ${ }^{15}$ o qual constataram que parte da população não possui conhecimento sobre o fato das mudanças de hábitos de vida interferir diretamente no processo saúde-doença. Ainda ressaltou que, estratégias tradicionais são pouco eficazes, e que há necessidade de novas formas para conscientizar o indivíduo/coletivo sobre mudanças comportamentais em prol da saúde. ${ }^{15}$

As atividades em grupo e palestras foram as ferramentas mais utilizadas nas estratégias abordadas nas ESFs e, devido a sua importância de aceitabilidade e participação da população, devem ser amplamente exploradas pelas equipes de saúde, através de esclarecimentos sobre alimentação saudável, cuidados bucais, importância de realizar exercício físico, cuidados com medicamentos (ingestão, armazenamento, etc.), e orientações que visam minimizar possíveis danos à saúde. ${ }^{16,17}$ Garcia et al. ${ }^{18}$ mostram que, para população idosa atendida nas Unidades Básicas de Saúde, podem ser disponibilizadas dinâmicas direcionadas para cada grupo/serviço através de palestras, diálogos, passeios, práticas corporais e grupos de trabalhos manuais (artesanato), como forma de promover a saúde.

Quando observadas as atividades desenvolvidas no âmbito escolar, nota-se a importância de capacitar o corpo docente para atuar em conjunto com a equipe de saúde, e abordar assuntos de educação em saúde, promoção e prevenção, bem como atuar diretamente com os escolares no intuito de conscientizá-los sobre hábitos saudáveis e os malefícios advindos do abuso de bebida alcoólica, drogas ilícitas e prática sexual sem proteção. ${ }^{11} \mathrm{Em}$ contrapartida, estudos mostram a dificuldade da equipe de saúde em desenvolver seu trabaIho através do Programa Saúde na Escola, uma vez que o acúmulo de demandas sobrecarrega as equipes das ESFs, fazendo com que as mesmas percam o interesse em atuar neste ambiente; e, ainda sugerem a criação de outra equipe integrada à ESF para atuar somente nas escolas, visando uma atenção primária adequada para estas crianças e adolescentes. ${ }^{19,20}$

Por fim, os achados demonstram que as estratégias utilizadas pelas ESFs contemplam o que está preconizado como "temas prioritários" na PNPS, cujo objetivo principal é expandir as estratégias e ações voltadas à promoção da saúde, nos diversos níveis do SUS. ${ }^{2}$ Desta maneira, é possível observar o avanço e a importância de institucionalizar práticas voltadas à estratégia de promoção da saúde, apesar de ser um tema que ainda está em processo de construção. ${ }^{21}$

\section{CONSIDERACÕ̃ES FINAIS}

A partir das leituras, nota-se a aproximação dos termos "promoção de saúde" e "prevenção de doenças" e, a dificuldade de selecionar estratégias voltadas para o tema pesquisado. Promover saúde é bem mais amplo do que prevenir doenças, pois a promoção visa identificar e enfrentar os determinantes do processo saúde-doença, transformando-os em favor da saúde.

Considerando o que é preconizado na PNPS, observa-se que nos artigos incluídos neste estudo a maioria das equipes multiprofissionais nas ESFs atua de maneira efetiva em ações que proporcionam saúde e qualidade de vida à população atendida, buscando orientá-las e capacitá-las quanto ao seu estilo de vida. Em alguns estudos houve oferta continuada ou por um período específico de ações/capacitações para população, enquanto que um estudo identificou baixa adesão na participação da comunidade, o que evidencia a necessidade de buscar novas ferramentas e metodologias mais atrativas e viáveis. Houve também casos que descrevem carência em relação as ações de promoção da saúde prestadas à população, que demonstra a importância da educação e formação continuada das equipes de saúde.

As ESFs desenvolvem ações orientativas, porém nota-se a deficiência de atividades práticas e oficinas que ampliem a participação dos sujeitos no processo de cuidado à saúde. Através dessas práticas, os sujeitos participam como atores na construção de saberes e práticas que visam favorecer as condições de saúde e, consequentemente, a autonomia no processo de escoIha e possibilidades para melhorar o estilo de vida.

Por fim, é de suma importância que as ESFs preconizem estratégias de promoção da saúde para a área geográfica pela qual é responsável, e divulguem os resultados dos trabalhos desenvolvidos. Através da publicação de artigos que contemplem estratégias e/ou ações realizadas, pode-se mostrar a atuação da equipe multiprofissional junto a população, além de apresentar o sucesso e fragilidades das atividades. Dessa maneira, possibilita que toda a rede de atenção primária à saúde consiga reproduzir, adequar às diferentes realidades ou, até mesmo, melhorar as estratégias implementadas.

\section{REFERÊNCIAS}

1. Brasil. Ministério da Saúde. Secretaria de Vigilância em Saúde. Secretaria de Atenção à Saúde. Política Nacional de Promoção da Saúde - 3. ed. - Brasília: Ministério da Saúde 
(Série B. Textos Básicos de Saúde), 2010.

2. Brasil. Ministério da Saúde. Secretaria de Vigilância em Saúde. Secretaria de Atenção à Saúde. Política Nacional de Promoção da Saúde: PNPS: revisão da Portaria MS/GM n 687, de 30 de março de 2006. Brasília: Ministério da Saúde, 2015.

3. Brasil. Ministério da Saúde. Secretaria de Atenção à Saúde. Departamento de Atenção Básica. Política Nacional de Atenção Básica. Brasília, DF, Ministério da Saúde, 2012.

4. Norman $A H$, Tesser CD. Acesso ao cuidado na Estratégia Saúde da Família: equilíbrio entre demanda espontânea e prevenção/promoção da saúde. Saúde Soc 2015;24(1):16579. doi: 10.1590/S0104-12902015000100013

5. Dias MSA, Parente JRF, Vasconcelos MIO, Dias FAC. Intersetorialidade e Estratégia Saúde da Família: tudo ou quase nada a ver? Ciênc. saúde coletiva 2014;19(11):437182. doi: 10.1590/1413-812320141911.11442014

6. Ferreira Neto JL, Oliveira GL, Viana NO, Duarte LGMF. Integralidade, condições de oferta de serviços e processo de trabalho de Equipes de Saúde da Família em Belo Horizonte. Saúde Debate 2016;40(111):179-192. doi: 10.1590/01031104201611114

7. Vieira RS, Vieira RS. Saúde do idoso e execução da política nacional da pessoa idosa nas ações realizadas na atenção Básica à Saúde. R. Dir. Sanit 2016;17(1):14-37.

8. Leite RS, Santos APM, Lima CA, Ribeiro CDAL, Brito MFSF. Estratégia Saúde da Família versus centro de saúde: modalidades de serviços na percepção do usuário. Cad. Saúde Colet 2016;24(3)323-29. doi: 10.1590/1414 462X201600030149

9. Silva SS, Mambrini JVM, Turci MA, Macinko J, Lima-Costa MF. Uso de serviços de saúde por diabéticos cobertos por plano privado em comparação aos usuários do Sistema Único de Saúde no Município de Belo Horizonte, Minas Gerais, Brasil. Cad Saúde Pública 2016;32(10):e00014615. doi: 10.1590/0102-311X00014615

10. Lindemann IL, Mendoza-Sassi RA. Orientação para alimentação saudável e fatores associados entre usuários da atenção primária à saúde no sul do Brasil. Rev Bras Promoç Saúde 2016;29(1):34-42. doi: 10.5020/18061230

11. Machado MFAS, Gubert FA, Meyer APGFV, Sampaio YPCC, Dias MSA, Almeida AMB, Morais APP, Silva AC, Campos JS, Chagas MIO, Chaves ES. Programa Saúde na Escola: estratégia promotora de saúde na atenção básica no Brasil.
J. Hum. Growth Dev 2015;25(3):307-312. doi: 10.7322/ jhgd.96709

12. Pasqual KK, Carvalhaes MABL, Parada CMG. Atenção à saúde da mulher após os 50 anos: vulnerabilidade programática na Estratégia Saúde da Família. Rev Gaúcha Enferm 2015;36(2)21-27. doi: 10.1590/19831447.2015.02.44822

13. Heidemann ITSB, Boehs AE, Fernandes GCM, Wosny AM, Marchi JG. Promoção da saúde e qualidade de vida: concepções da carta de Ottawa em produção científica. Cienc Cuid Saude 2012;11(3):613-619. doi: 10.4025/ cienccuidsaude.v11i3.13554

14. Durand M, Heidemann ITSB. Promoção da autonomia da mulher na consulta de enfermagem em saúde da família. Rev Esc Enferm USP 2013;47(2):288-295.

15. Costa JA, Balga RSM, Alfenas RCG, Cotta RMM. Promoção da saúde e diabetes: discutindo a adesão e a motivação de indivíduos diabéticos participantes de programas de saúde. Ciênc. saúde coletiva 2011;16(3):2001-2009. doi: 10.1590/ S1413-81232011000300034

16. Cavalcante PMT, Freitas MC. Em busca de um viver saudável: relato de experiência com um grupo de idosos cadastrados na estratégia saúde da família de Pacatuba - CE. Rev. RENE 2007;8(1):92-99.

17. Silva $A B$, Oliveira JL, Magalhães JM, Sales MCV. A assistência do enfermeiro da atenção básica ao adolescente com dependência química. R. Interd 2014;7(4):61-71.

18. Garcia MAA, Yagi GH, Souza CS, Odoni APC, Frigério RM, Merlin SS. Atenção à saúde em grupos sob a perspectiva dos idosos. Rev Latino-am Enfermagem 2006;14(2):175-82.

19. Donato LMTM, Moraes SHM, Nunes CB, Gerk MAS. A interação entre ESF e escola na saúde do adolescente. Cadernos ABEM 2012;8(1):13-19.

20. Couto NA, Kleinpaul WV, Borfe L, Vargas SC, Pohl HH, Krug SBF. O ambiente escolar e as ações de promoção da saúde. Cinergis 2016;17(4)378-383. doi: 10.17058/cinergis. v17i0.8150

21. Malta D, Silva MMA, Albuquerque GM, Amorin RCA, Rodrigues GBA, Silva TS, Jaime PC. Política Nacional de Promoção da Saúde, descrição da implementação do eixo atividade física e práticas corporais, 2006 a 2014. Rev Bras Ativ Fis Saúde 2014;19(3):286-299. doi: 10.12820/ rbafs.v.19n3p286 